IPM-94-054

\title{
Baryogenesis from Long Cosmic Strings
}

\author{
Masoud Mohazzab" \\ Institute for Studies in Theoretical Physics and Mathematics, \\ P.O.Box 5746, Tehran 19395, Iran.
}

\begin{abstract}
Based on the mechanism of cusp production on long cosmic strings, the baryon asymmetry caused by cusp annihilation has been calculated. The result is compatible with observation and stronger than the results from loops.
\end{abstract}

\footnotetext{
${ }^{1}$ e-mail addresses masoud@netware2.ipm.ac.ir and masoud@irearn.bitnet
} 


\section{Introduction}

Grand Unified Theories (GUT) are possible frameworks to explain the baryon asymmetry of the universe where the Sakharov's conditions [1] i.e. 1) baryon number violation, 2) C and CP violation and 3) departure from thermal equilibrium are satisfied. GUTs predict that the universe underwent a series of phase transitions during its early stage of evolution. Cosmic strings are one dimensional topological defects that are generated during the phase transition [2], [3]. Cosmic strings have to be in the form of loops or infinitely long with the important parameter $\mu$, mass per length of the strings and are very desirable in cosmology. The value $G \mu \sim 10^{-6}$ is compatible with

1) the scale of GUT symmetry breaking

2) structure formation models and

3) thermal fluctuations of the background radiation (the recent COBE results).

The oscillations of cosmic strings typically lead to the formation of cusps. A cusp is a point where two segments of the strings overlap and the point reaches the speed of light. There is no topological barrier to conserve cusps from decaying into bursts of Higgs or superheavy particles. The rate of decay and total energy of cusp annihilation has been worked out in [4] and [5], respectively. The starting point for the perturbative calculation is to consider the interacting lagrangian as [3]

$$
\mathcal{L}_{I}=\lambda|\phi|^{2} \psi^{2}
$$

where $\lambda$ is the coupling constant of the Mexican hat potential

$$
U(\phi)=\frac{1}{4} \lambda\left(|\phi|^{2}-\sigma^{2}\right)^{2}
$$

$\psi$ is the outgoing particles, $\sigma$ is the scale of GUT symmetry breaking and $\phi$ is the complex field configuration of the string.

The dimensionless value that most authors try to derive is the ratio of the net baryon number density, denoted by $n_{B}-n_{\bar{B}}$, to the entropy density of the universe, $s$, or $\eta=\frac{n_{B}-n_{\bar{B}}}{s}$. The observational value for $\eta$ based on the measurments of aboundances of primordial $\mathrm{D}$, ${ }^{3} \mathrm{He}$ and ${ }^{7} \mathrm{Li}$ is in the range $(6-10) \times 10^{-11}$.

Cusp annihilation on cosmic strings has long been considered as a mechanism to describe the baryon asymmetry of the universe [6]. The annihilation of cusps produce superheavy $\psi$ particles. Furthere decay of these particles results in the baryon asymmetry of $\Delta B \sim$ $10^{-2}-10^{-13}$ [0]. In [6] loops of ordinary cosmic strings are considered and the baryon number production from kinks and cusps evaporation has been calculated. The result of [6] is independent of era shows cusp annihilation on loops results in a compatible value for $\eta$. Kink evaporation, on the other hand, cannot describe the observed value of baryon asymmetry. Another mechanism to be accounted for baryogenesis is the collapse of the 
topological defects such as cosmic string loops or textures [8]. In the former approach, oscillations of cosmic string loops reduce their radius due to gravitational radiation. The

loops shrink to their minimum size $\sim \lambda^{-\frac{1}{2}} \sigma^{-1}$ when they have zero winding number and can decay into superheavy particles. The decay of these superheavy particles are accounted for the baryon number asymmetry. The other mechanism of [8] is the shrinking of the loops by cusp annihilation. In each period of oscillation of a loop, equivalent to its length, a cusp forms and radiates causing the loss of energy and therefore the decrease of the radius of the loop.

The ratios of baryon number generation of loops due to the decrease of its radius by gravitational radiation and cusp annihilation are respectively

$$
\begin{gathered}
\eta_{\text {g.r. }}=\eta_{\max }(G \mu)^{-\frac{3}{4}}\left(\frac{T_{F}}{T_{c}}\right)^{3} \\
\eta_{\text {c.a. }}=\eta_{\max } \lambda^{\frac{1}{2}}\left(\frac{T_{F}}{T_{c}}\right)^{3 / 2}
\end{gathered}
$$

where $\eta_{\max } \simeq .03 \frac{N_{x}}{N} \Delta B\left(10^{-13}<\Delta B<10^{-2}\right)$ and $N_{x}$ is the helicity of spin and $\mathrm{N}$ is the number of states. $T_{F}$ is the temperature at the time $t_{F}=(\gamma G \mu)^{-1} t_{F}^{\prime}$ where $t_{F}$ is the time that loops reaches to its minimum size length and decays and $t_{F}^{\prime}$ is the time when the loop is formed.

In [9] a mechanism has been suggested that produces cusps from colliding traveling waves on long strings. In this work, based on the same mechanism, we calculate the baryon asymmetry caused by long cosmic strings from cusp annihilation.

In the next section, we calculate the amount of baryon number from cusp annihilation on long strings.

\section{Baryon number production from long strings}

According to a numerical simulation, long strings are $80 \%$ of the total cosmic strings formed at the GUT phase transition [3]. Therefore they may have important contribution to radiation from cosmic strings.

Long strings do not shrink to a point and the only possibility for them to radiate superheavy particles is through cusp annihilation. Cusps, on long strings, are formed, up to a probability, when two wiggles traveling along the string collide. If we assume the wiggles have random shapes we can find the probability of formation of a cusp is $50 \%$ [9]. The probability of formation of more than one cusp in each collision is also non-zero. Of course it is clear that the shapes of wiggles are not random since the fractal dimension of cosmic strings is 1.2 (and not 2 for the random shape) [10]. Due to this mechanism and the possibility of 
superheavy particle production from cusps annihilation, long strings could contribute to the baryon number generation.

The scaling distribution of long strings is given by [3]

$$
n_{l . s .}^{\text {scaling }}=\frac{\nu}{t^{3}}
$$

where $\nu$ is a constant of the order 100 and $t$ is the cosmological time. The distribution before the scaling solution, however, is different and is given by [11]

$$
n_{l . s .}=\frac{\nu^{\prime}}{(G \mu)^{\frac{3}{2}} m_{p l}^{\frac{3}{4}} t^{3+\frac{3}{4}}}
$$

where $\nu^{\prime}$ is constant.

The distribution of traveling waves on long strings can roughly be written [9] as

$$
K(l, t)=\alpha \frac{t}{l^{2}}
$$

where $l$ is the size (the order of wavelength) of the traveling wiggle and $\alpha$ is a constant.

The total number of cusps per volume will be

$$
n_{c}(t)=P_{c} \int_{t_{\min }}^{t} d t^{\prime} n_{l . s .} \int_{l_{\min }}^{t^{\prime}} d l K\left(l, t^{\prime}\right) \frac{1}{l} z\left(t^{\prime}\right)^{-3}
$$

where $z(t)$ is the red shift factor at the time $t, \frac{1}{l}$ represents the frequency of the impacts of the wiggles. The probability of cusp formation on long string upon each collision is denoted by $P_{c}$.

In the integration (2.8) $l_{\min }$ is a minimum for the size of traveling waves, beyond which gravitational radiation smoothes out the wiggle

$$
l_{\min } \simeq \gamma G \mu t
$$

where $\gamma \simeq 100$

The minimum time $t_{\text {min }}$ is close to the time when cosmic string is formed, i.e. $t_{c}$, the time when GUT phase transition occurs.

It should be noted that we have neglected the effect of friction and the fact that the traveling waves do not change their shape when they travel along the string [12].

To implement the third Sakharov condition, i.e. out of equilibrium condition, we should have

$$
\Gamma \leq H
$$

where $\Gamma$ is the decay rate of the superheavy particle and $H$ is the expansion rate of the Universe. Here we consider the decay of superheavy particles and don't consider the inverse 
decay or collisions. Therefore the minimum time can be approximated by $t_{\text {min }}=t_{s} \sim 10 m_{\psi}^{-1}$ [6], when the inverse decay is suppressed by boltzman factor. $t_{s}$ is given by

$$
t_{s}=100 \sqrt{\frac{45}{16 \pi^{3} g_{*}}}\left(\frac{m_{\psi}}{m_{p l}}\right)^{-1} t_{p l}
$$

Using the number density (2.6), the total number of cusps at the time $t$, before the scaling solution era, will be

$$
n_{c}(t)=\frac{7 \nu^{\prime} P_{c} \alpha}{2(\gamma G \mu)^{2}(G \mu)^{\frac{3}{2}} m_{p l}^{\frac{3}{4}}} \frac{1}{t^{2}} \frac{1}{t_{s}^{\frac{7}{4}}}
$$

The net baryon number from cusp evaporation is

$$
n_{B}=n_{c} \Delta B
$$

where $\Delta B$ is the amount of baryon number violation due to $\mathrm{CP}$ violation from the decay of the superheavy particles and is in the range of $10^{-2}-10^{-13}$, depending on the model [7].

The baryon number asymmetry will then be

$$
\eta \equiv \frac{n_{B}}{s}=\frac{7 \nu^{\prime} P_{c} \alpha \Delta B}{2(\gamma G \mu)^{2}(G \mu)^{\frac{3}{2}}} \frac{1}{\left(.30118 g_{*}^{-\frac{1}{2}}\right)^{2}} \frac{1}{\frac{2}{45} \pi^{2} g_{* s}} \frac{T}{m_{p l}} \frac{1}{\left(m_{p l} t_{s}\right)^{\frac{7}{4}}}
$$

$s$ is the entropy density $s=\frac{2}{45} \pi^{2} g_{* s} T^{3}, g_{* s}$ is the number of states and $T$ is the temperature of the universe at the time $t$.

By (2.13) it is seen that the baryon asymmetry produced by long strings depends on the era. It is easy to see, however, that the asymmetry produced in the early era is much more important. In fact by using (2.5) instead of (2.6), in the integration (2.8), and performing the integration up to the present time it can be seen that the value of asymmetry is 30 order of magnitude less than the observed value. As a result we just consider the pre-scaling era. Another result of (2.13 is that the suppression factor ( i.e. the exponent of $T$ ) is 1 while in the case of loops it is more severe (equations (1.3) and (1.4) 8 ). For $\nu^{\prime} \sim 1, \gamma G \mu=10^{-4}$, $G \mu=10^{-6}, g_{*}=g_{* s}=100$ and using (2.11) we will have

$$
\eta=.566 \times 10^{11} \alpha P_{c} \Delta B \frac{T}{m_{p l}}\left(\frac{m_{\psi}}{m_{p l}}\right)^{\frac{7}{4}}
$$

The exact value for $P_{c}$ and $\alpha$ is not known yet, but it is surely $P_{c}<\frac{1}{2}$. In [9] it was assumed $\alpha=1$ which is an upper estimate for $\alpha$ and we should have $\alpha<1$.

Therefore, for $m_{\psi} \sim 10^{15} \mathrm{GeV}$ [5], $\alpha P_{c}=.5$ [9], $T \sim 10^{13} \mathrm{GeV}$ and $m_{p l} \sim 1.2 \times 10^{19} \mathrm{GeV}$ we see that for the value $\Delta B \sim 10^{-8}$, the baryogenesis from long cosmic strings is consistent with the observed value. 


\section{Conclusion}

Long strings are able to radiate superheavy particles via production and annihilation of cusps. Cusps may form on long strings when two traveling wiggles collide. The annihilation of cusps could be accounted for baryon asymmetry of the universe. By calculating the total number of cusps on long strings, as a function of time, we have calculated the amount of baryon asymmetry from them. It is assumed that one (or a pair of) superheavy gauge particle(s) is produced from each cusp annihilation. The suppression factor for long strings is less than that of loops. As a result long strings have more important contribution than loops to the baryon asymmetry.

\section{Acknowledgments}

I am grateful to Farhad Ardalan for useful comments.

\section{References}

[1] A. D. Sakharov, ZhETF Pis'ma 5 (1967) 32 [JETP Lett. 5 (1967) 24.]

[2] T. W. B. Kibble Phys. Rep. 67 (1980) 183.

[3] A. Vilenkin Phys. Rep. 121 (1985) 263.

[4] M. Serendicki and S. Theisen, Phys. Lett. B 189 (1987) 397; R. H. Brandenberger, Nucl. Phys. B 293 (1987) 812.

[5] M. Mohazzab, Int. J. Mod. Phys. D (1993).

[6] M. Kawasaki M. and K. Maeda Phys. Lett. B 209 (1988) 271.

[7] D. Nanopoulos and S. Weinberg, Phys. Rev. D 20 (1979) 2484; A. Yildiz and P. H. Cox, Phys. Rev. D 21 (1980) 906; P. Langacker in "CP violation" edited by C. Jorlskog, World Scientific, 1989.

[8] R. H. Brandenberger, A. C. Davis and M. Hindemarsh Phys. Lett. B 263 (1991) 239.

[9] M. Mohazzab and R.H. Brandenberger Int. J. Mod. Phys. D 2 (1993) 185.

[10] F. R. Bouchet, "The formation and evolution of cosmic strings", editted by G. Gibbons, S. Hawking and T. Vachespati, Cambridge University Press 1990; D. P. Bennett, ibid; N. Turok, ibid. 
[11] A. Everett, Phys. Rev.D 24 (1981) 858; T. W. B. Kibble, Acta Phys. Pol. B 13 (1982) 723.

[12] Vachespati and T. Vachespati Phys. Lett. B 238 (1990) 41; Grafinkle and T. Vachespati Phys. Rev. D 42, (1990) 1960. 